\title{
Postpartum Period in Women with Covid-19: Cytokine Storm and Coagulation System Changes, Points of View
}

\section{ISSN: 2640-9666}

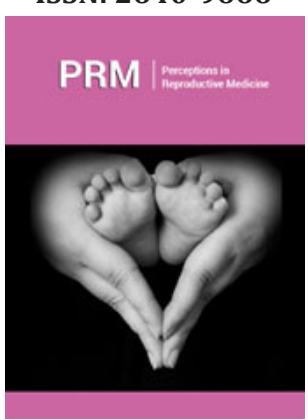

*Corresponding author: Maasoumeh Saleh, Department of Obstetrics and Gynecology, Iran

Submission: 漈 October 23, 2021

Published: 漈October 29, 2021

Volume 4 - Issue 5

How to cite this article: Sedigheh Hantoushzadeh, Maasoumeh Saleh, Mahboubeh Saleh, Behnaz Nouri. Postpartum Period in Women with Covid-19: Cytokine Storm and Coagulation System Changes, Points of View. Perceptions Reprod Med. 4(5). PRM. 000598. 2021.

DOI: $10.31031 /$ PRM.2021.04.000598

Copyright@ Saleh M, This article is distributed under the terms of the Creative Commons Attribution 4.0 International License, which permits unrestricted use and redistribution provided that the original author and source are credited.

\author{
Sedigheh Hantoushzadeh${ }^{1}$, Maasoumeh Saleh${ }^{1 *}$, Mahboubeh Saleh${ }^{2}$, Behnaz $^{2}$ \\ Nouri ${ }^{3}$ \\ ${ }^{1}$ Department of Obstetrics and Gynecology, Iran \\ ${ }^{2}$ Department of Urogynecology, Iran \\ ${ }^{3}$ Department of Community Medicine, Iran
}

\begin{abstract}
Patients with severe COVID-19 may progress to coagulopathy, which is characterized by thrombocytopenia, prolonged PT and aPTT, elevated D-dimer and decreased fibrinogen. Pregnancy itself is also a hypercoagulable state with higher risk of Venous Thromboembolism (VTE), especially after the placental expulsion and the postpartum period, where clotting activity is at its highest. Pregnancy is associated with almost 3 times the risk of thrombotic complications. Despite both acute and chronic complications of VTE, the importance and management of VTE in the context of COVID-19 infections in pregnant women has not been described well. This review was conducted to provide insight into pathophysiology of COVID-19 disease in the context of postpartum period and its clinical implications. Herein, we discuss the underlying pathogenesis of VTE and thrombotic incidences in patients with ongoing SARS-CoV-2 infections and provide strategies to limit morbidity and mortality in the management of these subset of patients in clinical settings.
\end{abstract}

Keywords: Coagulopathy; Thrombosis; SARS-CoV-2; Pregnancy; Parturition

\section{Introduction}

In the form of venous and arterial thromboembolism, coagulopathy is emerging as one of the most severe sequelae of the COVID-19 disease and has been prognostic of poorer outcomes [1-4]. Approximately $20 \%$ of patients with COVID-19 have severe coagulation abnormalities, and almost all severe and critically ill COVID-19 infection shows major coagulation disorders [5-7]. The majority of the cases of coagulopathy have been reported in patients with moderateto-severe COVID-19 and are limited to observations during the recovery/post-cytokine storm state [3]. Several pathways attempt to explain the mechanism behind the pro-coagulant state seen with COVID-19 patients, including different receptor binding, cytokine storm, and direct endothelial damage [3]. Normal pregnancy is accompanied by changes in the coagulation and fibrinolytic systems, including an increase in the majority of clotting factors and a decrease in anticoagulants, so pregnancy is a hypercoagulable state with a 4- to 6- fold increased risk of VTE. Multiple changes occur to the coagulation system as pregnancy progresses, with the most significant changes being seen at term gestation [8-10]. The increase in clotting activity is greatest when delivering placental expulsion, releasing thromboplastic substances, clotting, and stopping blood loss after delivery [11]. Admission of pregnant women to a hospital is associated with an 18-fold increased VTE risk sustained after discharge, especially for women older than 35 years, in the third trimester of pregnancy, and admitted for three days or longer [12].

Venous Thromboembolism (VTE) in pregnancy remains a leading cause of direct maternal mortality in the developed world, and identifiable risk factors increase in incidence [13]. In 
addition to mortality, VTE can cause chronic morbidity. Long-term morbidity, called a post-thrombotic syndrome, manifests as various degrees of edema, pain, and eczema, reduces life quality and is associated with substantial health care costs [14]. As pregnancy is already a physiologically hypercoagulable state, it seems likely that pregnant women with COVID-19 would be at especially high risk of VTE. Thromboembolic complications have been reported in $0.28 \%$ of pregnant women with COVID-19, but it is $0.1 \%$ in women without COVID-19 [15]. The most common pattern of coagulopathy observed in patients hospitalized with COVID-19 is characterized by elevations in fibrinogen and D-dimer levels and mild prolongation of PT/aPTT. This correlates with a parallel rise in markers of inflammation (e.g., CRP). Unlike the pattern seen in classic Disseminated Intravascular Coagulation (DIC) from bacterial sepsis or trauma, prolongation of the PTT and PT is minimal, thrombocytopenia is mild, and lab results supporting microangiopathy are infrequent [16]. Patients with severe or critical COVID-19 are at risk of both immunothrombosis as well as hospital-associated VTE. Although thromboprophylaxis is wellestablished to prevent the risk of hospital-associated VTE, its role in preventing immunothrombosis remains uncertain [17]. Current advice from the RCOG recommends that all pregnant women admitted with confirmed or suspected COVID-19 receive prophylactic Low Molecular Weight Heparin (LMWH), unless birth is expected within 12 hours, and continue this for 10 days following discharge [18].

\section{Discussion}

The risk of VTE is highest during the first three weeks after delivery and remains elevated throughout the first 12 weeks after delivery because the prothrombotic changes do not revert completely normal until several weeks after delivery. The disturbed fibrinolysis becomes normal quickly as the placenta detached; it becomes similar to non-pregnant women in the first 24-48 hours after delivery [19]. The risk of pulmonary embolism is higher than Deep Vein Thrombosis (DVT) in postpartum. Thrombotic events increase after parturition and during the postpartum period of pregnant women with COVID-19; due to both the cytokine storm and the physiologic coagulation changes in pregnancy. Acute phase reactants (including CRP), fibrinogen, platelet counts, and antithrombin levels increase during the first week of the postpartum period. The D-dimer level also increases, reflecting the fibrinolysis increment. D-dimer is higher in severe COVID-19 patients and associated with an increased risk of VTE.

COVID-19 is a new and evolving disease and literature addressing the issue of thrombosis in pregnancy and the postpartum period is sparse, so we need more studies to achieve high-quality evidence. A prophylactic regimen for preventing venous thromboembolic events fails in $7.7 \%$ of COVID-19 cases admitted in ICU [20]. There is considerable uncertainty around the optimal duration of anticoagulants in the postpartum period after discharge. The decision depends on the severity of COVID-19 and other risk factors of VTE such as obesity, medical diseases, etc. In the postpartum period, for patients with recent COVID-19 infection, the benefits of thromboprophylaxis with LMWH outweigh the hemorrhagic risk. In addition to their anticoagulant properties, these drugs also have anti-inflammatory effects.

IL-6 level increases due to tissue trauma during surgery. Normal pregnancy is associated with a controlled inflammatory process, while Cesarean Section (CS), as major surgery, may aggravate the previously increased cytokine levels in pregnant women [21]. General anesthesia, as compared to spinal anesthesia, significantly increased the IL- 6 and TNF- $\alpha$ levels after CS. Cytokine levels also rise during and after vaginal delivery due to labor pain [22]. Pain during labor and perioperative pain may play an important role in modulating cytokines [23], so analgesic modalities can reduce cytokine storm. Also, cytokine levels will be different after emergency and elective CS.

Determination of specific cut-off values of coagulation and inflammatory parameters associated with adverse outcomes in the postpartum period is needed. The important point is to control inflammation, a risk factor for VTE; therefore, controlling inflammation by increasing the corticosteroid dose in the postpartum period is also wise. The anticoagulation and antiinflammatory agents' dose and administration duration adjustment following the parturition seems to be necessary. An important cause is that the postpartum period is a pro-inflammatory phase. This process is exacerbated in the postpartum period in the COVID-19 patients, and cytokine storms occur, especially after CS. An important issue is registering all the pregnant cases of COVID-19 and following them till 12 weeks after delivery to create guidelines ensuring an adequate level of care in the postpartum period.

\section{Conclusion}

We suggest that in addition to considering major and minor risk factors for VTE in the postpartum period based on previous guidelines, inflammatory risk factors (factors leading to cytokine storm such as painful and prolonged labor, CS, anesthesia especially GA, the rate of tissue trauma during vaginal delivery or CS, Operation duration, COVID-19 severity, phases of the disease in which the patient is, etc.) should also be included in VTE risk classification.

\section{References}

1. Zhou F, Yu T, Du R, Fan G, Liu Y, et al. (2020) Clinical course and risk factors for mortality of adult inpatients with COVID-19 in Wuhan, China: A retrospective cohort study. The Lancet 395(10229): 1054-1062.

2. Guan WJ, Ni ZY, Hu Y, Liang WH, Ou CQ, et al. (2020) Clinical characteristics of coronavirus disease 2019 in China. New England Journal of medicine 382(18): 1708-1720.

3. Huang C, Wang Y, Li X, Ren L, Zhao J, et al. (2020) Clinical features of patients infected with 2019 novel coronavirus in Wuhan, China. The Lancet 395(10223): 497-506.

4. Tang N, Li D, Wang X, Sun Z (2020) Abnormal coagulation parameters are associated with poor prognosis in patients with novel coronavirus pneumonia. Journal of Thrombosis and Haemostasis 18(4): 844-847.

5. Chan KH, Lim SL, Shaaban H, Guron G, Slim J (2021) Persistent hypercoagulable state in COVID-19: A case series of COVID-19 associated pulmonary embolism. Journal of Global Infectious Diseases 13(1): 38. 
6. Chen N, Zhou M, Dong X, Qu J, Gong F, et al. (2020) Epidemiological and clinical characteristics of 99 cases of 2019 novel coronavirus pneumonia in Wuhan, China: A descriptive study. The Lancet 395(10223): 507-513.

7. Han H, Yang L, Liu R, Liu F, Wu KL, et al. (2020) Prominent changes in blood coagulation of patients with SARS-CoV-2 infection. Clinical Chemistry and Laboratory Medicine (CCLM) 58(7): 1116-1120.

8. Alam W (2021) Hypercoagulability in COVID-19: A review of the potential mechanisms underlying clotting disorders. SAGE open medicine 9: 20503121211002996.

9. Brenner B (2004) Haemostatic changes in pregnancy. Thrombosis Research 114(5-6): 409-414.

10. James AH (2010) Pregnancy and thrombotic risk. Critical Care Medicine 38(2 Suppl): S57-S63.

11. Bremme KA (2003) Haemostatic changes in pregnancy. Best Practice \& Research Clinical Haematology 16(2): 153-168.

12. Sultan AA, West J, Tata LJ, Fleming KM, Piercy NC, et al. (2013) Risk of first venous thromboembolism in pregnant women in hospital: Population based cohort study from England. BMJ p. 347.

13. Simcox LE, Ormesher L, Tower C, Greer IA (2015) Pulmonary thromboembolism in pregnancy: Diagnosis and management. Breathe 11(4): 282-289.

14. Wik H, Jacobsen A, Sandvik L, Sandset P (2012) Prevalence and predictors for post-thrombotic syndrome 3 to 16 years after pregnancyrelated venous thrombosis: A population-based, cross-sectional, casecontrol study. Journal of Thrombosis and Haemostasis 10(5): 840-847.

15. Rabinovich A, Kadir AR, Thachil J, Iba T, Othman M, et al. (2019) DIC in obstetrics: Diagnostic score, highlights in management, and international registry-communication from the DIC and Women's Health
SSCs of the International Society of thrombosis and haemostasis. Journal of Thrombosis and Haemostasis 17(9): 1562-1566.

16. Lee AY, Connors J, Kreuziger LB, Murphy M, Gernsheimer T, et al. (2020) COVID-19 and coagulopathy: Frequently asked questions. American society of hematology/Covid-19 resources.

17. OBE BH, Retter A, McClintock C (2020) Practical guidance for the prevention of thrombosis and management of coagulopathy and disseminated intravascular coagulation of patients infected with COVID-19.

18. RCOG (2020) Coronavirus (COVID-19) Infection in pregnancy: Information for health care professionals London: Royal College of Obstetricians and Gynaecologists. London, UK.

19. Hellgren M (2003) Hemostasis during normal pregnancy and puerperium. Seminars in thrombosis and hemostasis 29(2): 125-130.

20. Klok F, Kruip M, Vander MN, Arbous M, Gommers D, et al. (2020) Incidence of thrombotic complications in critically Ill ICU patients with COVID-19. Thromb Res 191: 145-147.

21. Takacs P, Green KL, Nikaeo A, Kauma SW (2003) Increased vascular endothelial cell production of interleukin-6 in severe preeclampsia. American Journal of Obstetrics and Gynecology 188(3): 740-744.

22. Puchner MA, Protonotariou E, Boutsikou T, Makrakis E, Sarandakou A, et al. (2005) The influence of the mode of delivery on circulating cytokine concentrations in the perinatal period. Early Human Development 81(4): 387-392.

23. Barr J, Boulind C, Foster J, Ewings P, Reid J, et al. (2015) Impact of analgesic modality on stress response following laparoscopic colorectal surgery: a post-hoc analysis of a randomised controlled trial. Techniques in coloproctology 19(4): 231-239.

For possible submissions Click below: 